\title{
Effects of a Two-Week, Hospitalized Phase II Cardiac Rehabilitation Program on Physical Capacity, Lipid Profiles and Psychological Variables in Patients With Acute Myocardial Infarction
}

\author{
Toshiko Yoshida, $\mathrm{PhD} *, * * ;$ Kazunori Yoshida, MD*; Chitose Yamamoto, MS*; \\ Makoto Nagasaka, MD*; Hiroko Tadaura, RN*; Taiichiro Meguro, MD***; \\ Tokutaro Sato, $\mathrm{MD}^{\dagger}$; Masahiro Kohzuki, MD*
}

\begin{abstract}
A new 2-week hospitalized phase II cardiac rehabilitation program has been designed and the present study sought to clarify whether the physical and psychological status of patients with acute myocardial infarction (AMI) improved after participation in the program. Fifty-one patients with AMI were enrolled in the rehabilitation program, which consisted of exercise training, education and counselling, and another 34 patients with AMI who did not participate in the program served as the control group. The physical and psychological status of the patients was evaluated before, at 1-month after the program, and at 6- and 12-months follow-ups. The physical status was assessed by exercise tolerance and serum lipid profiles and the psychological status was assessed by the Spielberger State-Trait anxiety inventory questionnaire (STAI) and self-rating questionnaire for depression. Quality of life (QOL) was assessed using established and validated QOL scales. After participation in the program, the exercise tolerance, serum lipid profiles and STAI anxiety score of the patients were improved significantly and at the 6month follow-up these parameters remained improved and regular physical activity was maintained. The QOL score also improved significantly. Even at the 12-month follow-up, lipid profiles remained improved and regular physical activity was maintained. The 2-week hospitalized phase II cardiac rehabilitation program improved the management of cardiac risk factors and psychological status in patients with myocardial infarction (MI). It provides beneficial effects on the patient's physical and psychological activities in the recovery phase and may also contribute to the secondary prevention of MI. (Jpn Circ J 2001; 65: 87-93)
\end{abstract}

Key Words: Cardiac rehabilitation; Exercise; Myocardial infarction; Quality of life

$\mathbf{T}$ he goal of cardiac rehabilitation is to ensure the best possible physical, psychological and social conditions for patients with chronic or post-acute cardiac disease so that they may, by their own efforts, preserve or resume their proper place in the society! The program usually consists of 3 stages: the acute stage (phase I), subacute stage (phase II) and maintenance stage (phase III), which include exercise training, medical surveillance, emergency support, and psychological and vocational counselling. The period of hospitalization during phase I is becoming shorter as a result of recent advances in medical interventions and for economic reasons. Therefore, lifestyle modification, particularly in terms of daily exercise, cessation of smoking and a balanced food intake, should be acquired during phase II and maintained thereafter, but only $11-38 \%$ of patients with coronary heart disease participate in phase II cardiac rehabilitation programs ${ }^{2,3}$ In Japan, particularly, almost all cardiac rehabilitation programs

(Received June 15, 2000; revised manuscript received November 1, 2000; accepted November 6, 2000)

*Department of Internal Medicine and Rehabilitation Science, Tohoku University Graduate School of Medicine, Sendai, **Miyagi University, Sendai, ***Sendai Kousei Hospital, Sendai and ${ }^{\dagger}$ National Rehabilitation Center for the Disabled, Tokorozawa, Japan

Mailing address: Professor Masahiro Kohzuki, MD, Department of Internal Medicine and Rehabilitation Science, Tohoku University Graduate School of Medicine, Sendai 980-8574, Japan. E-mail: kohzuki@mail.cc.tohoku.ac.jp consist of phase I only and phase II cardiac rehabilitation is utilized by less than $1 \%$ of patients.,5 It is usually initiated as an outpatient program within 2-3 weeks of hospital discharge and requires patients to visit local hospitals 2-3 times per week for 3-6 months, which many patients find difficult to do. Furthermore, only few hospitals in Japan offer a phase II rehabilitation program and almost none offer it in the evening. Therefore it is difficult for patients to return to full work as soon as possible with confidence after myocardial infarction (MI). To solve these problems, we initially designed a 4-week hospitalized phase II cardiac rehabilitation program and demonstrated considerable improvement in the physical and psychological status of patients with MI6 We reviewed that program and have now designed a 2-week hospitalized phase II cardiac rehabilitation program, which we consider is sufficient time to supervise exercise training and for the patient to gain a better understanding of life style modification.

The purpose of the present study was to clarify whether the physical and psychological status of patients with MI improved after participation in the new program and to compared the effects of the 2-week rehabilitation program with those of the 4-week program.

\section{Methods}

Subjects

Fifty-one patients (47 men, 4 women; age, 25-70 years) 
Table 1 Baseline Characteristics of the Study Groups

\begin{tabular}{|c|c|c|c|c|}
\hline & $\begin{array}{c}2 \text {-week } \\
\text { rehabilitation }\end{array}$ & $\begin{array}{c}\text { 4-week } \\
\text { rehabilitation }\end{array}$ & Control & $p$ value \\
\hline$n$ & 51 & 29 & 34 & \\
\hline Age (years) & $52.0 \pm 10.1$ & $57.4 \pm 10.5$ & $61.5 \pm 10.7$ & 0.0003 \\
\hline Male & $47(92 \%)$ & $27(93 \%)$ & $27(97 \%)$ & $N S$ \\
\hline Peak CK (IU/L) & $2785 \pm 2164$ & $2593 \pm 1943$ & $2476 \pm 2132$ & NS \\
\hline Location of infarction & & & & NS \\
\hline Anterior wall & $24(47 \%)$ & $17(59 \%)$ & $14(42 \%)$ & \\
\hline Inferior wall & $18(35 \%)$ & $7(24 \%)$ & $17(52 \%)$ & \\
\hline Other & $9(18 \%)$ & $5(17 \%)$ & $2(6 \%)$ & \\
\hline \multicolumn{5}{|l|}{ Intervention } \\
\hline Coronary stenting & $35(67 \%)$ & $11(38 \%)$ & $18(53 \%)$ & 0.03 \\
\hline Medication & & & & $N S$ \\
\hline ACE inhibitors & $38(76 \%)$ & $15(54 \%)$ & $24(71 \%)$ & \\
\hline$\beta$-blockers & $4(8 \%)$ & $0(0 \%)$ & $0(0 \%)$ & \\
\hline Lipid lowering agents & $17(33 \%)$ & $7(24 \%)$ & $7(22 \%)$ & \\
\hline$B M I\left(k g / m^{2}\right)$ & $24.4 \pm 3.0$ & $24.5 \pm 2.6$ & $25.5 \pm 3.1$ & $N S$ \\
\hline Hypertension & $26(51 \%)$ & $13(45 \%)$ & $23(68 \%)$ & $N S$ \\
\hline Diabetes & $12(24 \%)$ & $8(24 \%)$ & $9(26 \%)$ & $N S$ \\
\hline Hyperlipidemia & $36(71 \%)$ & $17(59 \%)$ & $18(53 \%)$ & $N S$ \\
\hline Hyperuricemia & $12(24 \%)$ & $4(14 \%)$ & $7(21 \%)$ & $N S$ \\
\hline Smoking habit & $47(92 \%)$ & $25(86 \%)$ & $24(71 \%)$ & 0.009 \\
\hline
\end{tabular}

Values are expressed as the number of patients or mean $\pm S D$. Overall significant difference was analyzed by one-way ANOVA with Fisher's probability least significant difference test. CK, creatine kinase; BMI, body mass index; NS, no significant difference.

with MI enrolled in the program. They underwent coronary interventions, such as plain old balloon angioplasty (POBA), coronary stenting or percutaneous transluminal coronary recanalization (PTCR), approximately $2-3$ weeks before entry into the program and were referred by their attending physicians. Another 34 patients (27 men, 7 women; age, 39-82 years) with MI who underwent coronary interventions, but who did not participate in the rehabilitation program, served as a control group. Twenty-nine patients with MI had enrolled in the 4-week phase II rehabilitation program previously6 All patients were classified as New York Heart Association functional class I without signs of overt congestive heart failure. We excluded patients with other serious diseases (eg, renal failure, cancer) at the baseline (Table 1). Except for the rehabilitation protocol, the standard medical care during the follow-up period was similar for all patients and comprised a routine cardiological checkup every 3 to 6 months. All patients received 81-162 $\mathrm{mg}$ of aspirin and almost all patients took isosorbide dinitrate. Angiotensin-converting enzyme inhibitors were prescribed for 38 patients $(55 \%)$ on the 2-week program, 15 patients (52\%) on the 4-week program and 24 patients $(71 \%)$ in the control group, and lipid-lowering agents (simvastatin, pravastatin or fibrate) administered to 21 patients $(41 \%)$ on the 2 -week program and 7 patients $(24 \%)$ on the 4-week program; the doses were unchanged during the study period.

The study protocol was approved by the institional committee and all patients gave informed consent.

\section{Rehabilitation Protocol}

The rehabilitation program consisted of 3 elements: supervised exercise training, under prescription and based on cardiopulmonary treadmill exercise testing, education and counselling for patients and their families. The exercise training consisted of stretching, 30-40 min of exercise on a bicycle ergometer, which included a warm-up and cooldown, under the supervision of a physiotherapist twice a day, and 20-30 min of self-monitored walking 2-3 times a day, 7 days a week. The latter 2 types of exercises were performed at $80-100 \%$ of heart rate (HR) at the anaerobic threshold (AT) determined by treadmill testing? In order to prevent coronary thrombosis, patients who had undergone coronary stenting were not allowed to undergo treadmill testing for at least 8 weeks after the intervention? Instead we first prescribed individual exercise programs with an exercise HR equal to the resting heart rate plus 20 and the patient was evaluated for exercise tolerance 8 weeks after the intervention. They were then given exercise programs with an intensity of $80-100 \%$ of HR at the AT thereafter.

The objective of patient education is information exchange and health education. Regular educational sessions, using either a lecture, given by doctors, nurses, nutritionists or physiotherapists, or video format, were given to small groups and consisted of 7 classes: knowledge of ischemic heart disease; risk factor modification, including smoking cessation; physical training; management of diet; safe levels of activity during daily living; stress management; and return to work.

Individual counselling discussed specific aspects of the patient's diseases and physical and psychosocial conditions. Work tolerance was determined based on the results of the last treadmill test and progress in the exercise program. The final counselling session included recommendations and a decision about return to work.

After finishing this hospitalized program, the patients performed home-based and self-managed exercise training at $80-100 \%$ of $\mathrm{HR}$ at the AT.

\section{Evaluation}

We evaluated the physical parameters of patients who underwent POBA or PTCR in the rehabilitation groups before and 1 month after completion of the program and at the 6- and 12-month follow-ups. The physical parameters of those patients in the rehabilitation group who underwent coronary stenting were evaluated on at 1 month after the completion of the program and at the 6- and 12-month follow-ups.

The physical parameters were evaluation of exercise 
tolerance and serum lipid levels. The peak oxygen consumption (peak $\dot{\mathrm{V}} \mathrm{O}_{2}$ ) and AT of each patient were determined by progressive treadmill exercise testing. The test endpoint was the time when HR reached 190 beats/min minus each patient's age (target HR), or when the patient could not continue testing before reaching the target HR. The AT was determined by the 'V-slope' method ${ }^{9,10}$ using an expiratory gas analysis machine (Model 2900, Sensormedics, USA) coupled to an IBM computer. Fasting serum samples were obtained in the morning of the day after admission and the concentrations of triglyceride (TG), total cholesterol (TC) and high-density lipoprotein cholesterol (HDL-C) were determined with an auto-analyzer. Concentrations of lowdensity lipoprotein cholesterol (LDL-C) were calculated with the Friedewald equation using concentrations of TC, HDL-C and TG!1

In both rehabilitation groups and the control group, we evaluated the self-report questionnaire, which assessed the perceived physical activity and psychological status of anxiety and depression. Perceived physical activity was assessed by the frequency in 1 week of exercise lasting more than $20 \mathrm{~min}$. The patients were then classified into 3 groups: sedentary group, if they did not have any physical activity; low physical activity group, if they had physical activity less tha twice a week; and regular physical activity group, if they had physical activity equal to or more than twice per week. We evaluated these parameters in both rehabilitation groups and the control group before entry into the program and at the 6- and 12-month follow-ups.

Anxiety status was measured by Spielberger's StateTrait Anxiety Inventory Questionnaire (STAI)! ${ }^{12}$ Items for state anxiety were selected for their ability to discriminate between stress and non-stress conditions. The state anxiety score (STAI-I) consists of 20 statements on a 4-point scale covering apprehension, tension, nervousness, and worry, which evaluates how the subjects feeling 'at this moment'. The trait anxiety score (STAI-II) consists of 20 statements on a 4-point scale pertaining to how the subject generally feels. Both scales are designed to contain anxiety present and anxiety absent factors. To score, the 20 responses for each scale are summed ${ }^{13}$ and the total score for both ranges from 20 to 80, with a lower score reflecting a better psychological state. Depression status was measured by the SelfRating Questionnaire for Depression (SRQ-D) ${ }^{14}$ which evaluates mild and masked depressive conditions. The subjects tested have a choice of 4 answers to each question: seldom or never, some of the time, quite often or almost always. For any one question, these answers are scored 0,1 , 2 and 3, respectively. In scoring a completed questionnaire, the control items ( 6 items) are crossed out 15 and the score ranges from 0 to 36 , with a score of 11-16 representing borderline depression and a score above 16 representing depression! ${ }^{14}$ The reliability and validity of those questionnaires have been established and they have been used in a variety of clinical populations, including patients with cardiac disease! 13,14

Quality of life (QOL) was assessed using the established and validated QOL Score for Japanese Patients with Cardiovascular Diseases ${ }^{16}$ which consists of 39 items divided into the following 3 dimensions: subjective evaluation of health (8 items), social attitude and subjective wellbeing (21 items), and disease-specific conditions (10 items). The score ranges from 0 to 78 and a higher score means better QOL 15 The reliability and validity of those questionnaires have been established and they have been used in a variety of clinical populations! $13,14,16$

In the rehabilitation group, serum lipid levels were obtained and questionnaires were conducted at the baseline and at 1 month, 6 months and 12 months after completion of the program, except the patients who underwent POBA or PTCR who were evaluated on exercise tolerance at the baseline and at 1-, 6- and 12-months follow-ups; patients who underwent coronary stenting were evaluated on exercise tolerance at 1-, 6- and 12-months follow-ups. In the control group, we evaluated questionnaires at the baseline and at 6and 12-months follow-ups.

\section{Statistical Analysis}

Data were expressed as mean \pm SD. Student's t-test was used to compare differences between continuous variables. Differences between groups were examined by chi-square analysis or one-way analysis of variance (ANOVA). Significant differences were determined by Fisher's probability least significant difference test for post hoc comparisons. A $\mathrm{p}$ value less than 0.05 was considered significant.

\section{Results}

No patient in the rehabilitation group showed any symptoms, such as post-infarction angina, during the program. In the control group, 1 patient died of a recurrence of MI during the first 6-month follow-up period. No one died in the rehabilitation groups during the study period.

\section{Exercise Tolerance}

Patients with coronary stenting were evaluated for exercise tolerance 8 weeks after the intervention, so only 18 of the 51 patients in the 2-week rehabilitation group and 17 of the 29 patients in the 4-week rehabilitation group were examined for exercise tolerance before the commencement of the phase II rehabilitation program. In the 2-week rehabilitation group, the peak $\dot{\mathrm{V}}_{2}$ and AT had increased significantly 1 month after the program compared with before the program (Fig 1A,B). The patients with coronary stenting underwent treadmill testing 1 month after the 2-week program or just after the 4-week program. At 6 months after the program, peak $\dot{\mathrm{V}}_{2}$ had increased significantly compared with that of 1 month after the program in the 2week rehabilitation program (Fig 2A). However, the AT did not increase significantly in either group. When the peak $\dot{\mathrm{VO}}_{2}$ and AT were analyzed in patients who received all consecutive exercise tests, there were no significant differences in these parameters during the study period (repeated ANOVA). When 4 patients who were taking $\beta$ blockers were excluded from the analysis, the results did not change (data not shown).

\section{Serum Lipids}

Before commencement of the 2-week rehabilitation, a considerable percentage of patients had abnormal serum lipid concentrations: $12 \%$ for TC ( $\geq 220 \mathrm{mg} / \mathrm{dl}), 49 \%$ for TG $(\geq 150 \mathrm{mg} / \mathrm{dl}), 80 \%$ for HDL-C $(\leq 40 \mathrm{mg} / \mathrm{dl})$, and $14 \%$ for LDL-C $(\geq 120 \mathrm{mg} / \mathrm{dl})$. In the 2-week rehabilitation group, serum concentrations of TC and LDL-C did not change during the study period. Serum concentrations of TG and HDL-C, however, had improved significantly just after completion of the program, and at the 1-month, 6-month and 12-month follow-ups. In the 4-week rehabilitation group, serum concentrations of TG and HDL-C had improved significantly at the 6- and 12-month follow-ups. In addition, 
A

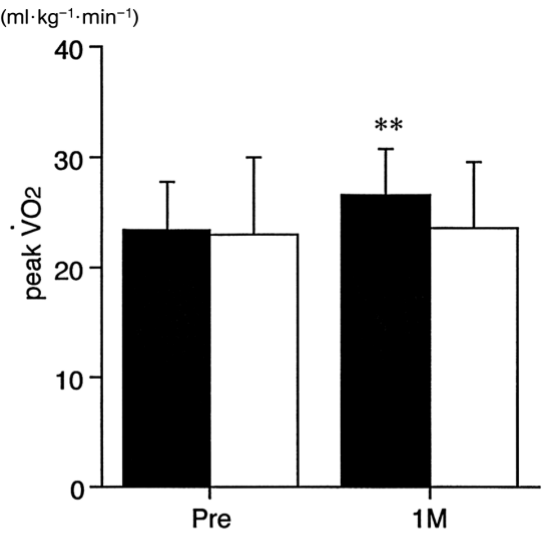

B

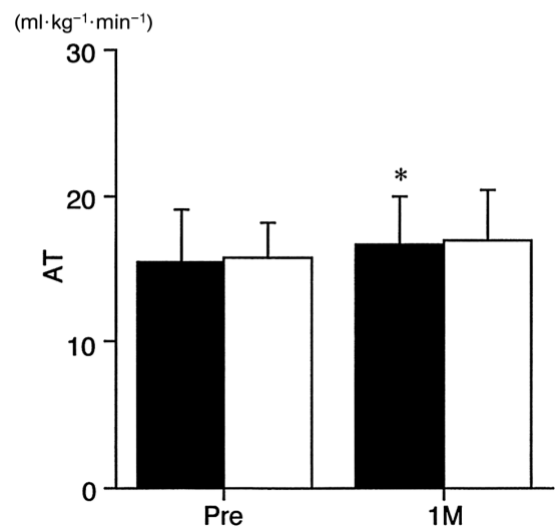

Fig 1. Exercise tolerances before rehabilitation (Pre) and at 1-month follow-up (1M) in patients who received plain old balloon angioplasty (POBA). (ם) 2-week rehabilitation group; ( $\square$ ) 4-week rehabilitation group; Peak $\dot{\mathrm{V}}$ 2, peak oxygen consumption; AT, anaerobic threshold. Data are expressed as mean \pm SD. $* \mathrm{p}<0.05, * * \mathrm{p}<0.01$ vs Pre.
A

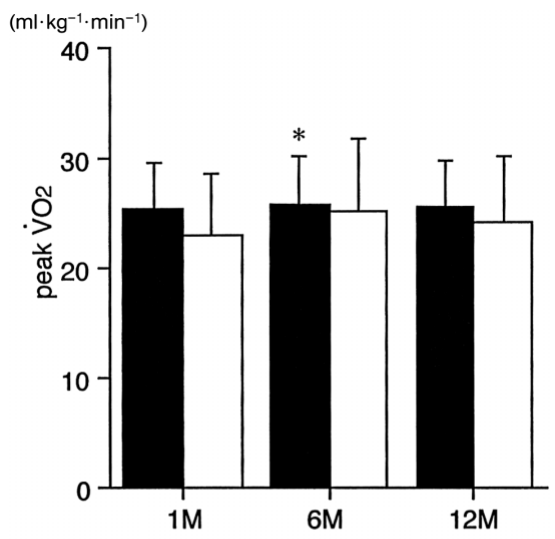

B

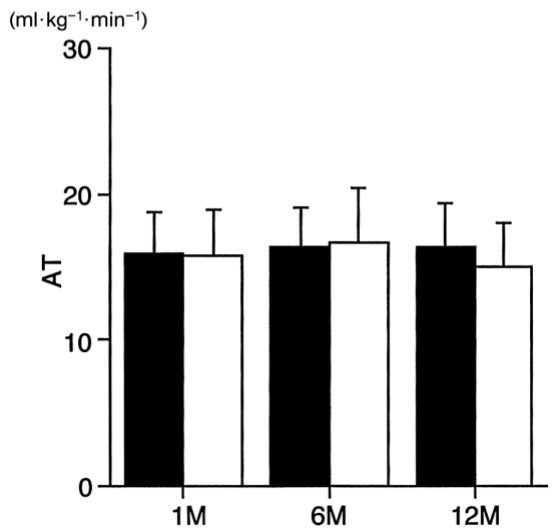

Fig 2. Exercise tolerances in the rehabilitation groups at 1-month (1M), 6-month (6M) and 12-month follow-up. (正) 2-week rehabilitation group; ( $\square$ ) 4-week rehabilitation group; Peak $\dot{\mathrm{V}}_{2}$, peak oxygen consumption; AT, anaerobic threshold. Data are expressed as mean \pm SD. ${ }^{*} \mathrm{p}<0.05$ vs $1 \mathrm{M}$.

Table 2 Serum Lipid Profiles at the Baseline and at 2-Week, 1-Month, 6-Month and 12-Month Follow-ups in Patients Who Enrolled in the 2-Week or 4-Week Rehabilitation Program

\begin{tabular}{|c|c|c|c|c|c|}
\hline & Baseline & 2-week & 1-month & 6-month & 12-month \\
\hline \multicolumn{6}{|l|}{ 2-week rehabilitation } \\
\hline$T C(m g / d l)$ & $182.5 \pm 33.8$ & $191.9 \pm 35.0 * *$ & $189.6 \pm 29.3$ & $188.6 \pm 36.4$ & $182.8 \pm 29.0$ \\
\hline$H D L-C(m g / d l)$ & $35.1 \pm 8.5$ & $38.3 \pm 8.0 * *$ & $44.6 \pm 9.2 * *$ & $50.8 \pm 12.2 * *$ & $51.2 \pm 10.5 * *$ \\
\hline$L D L-C(m g / d l)$ & $112.7 \pm 32.2$ & $124.2 \pm 30.7 * *$ & $119.8 \pm 29.2$ & $110.9 \pm 28.6$ & $105.5 \pm 24.5$ \\
\hline$T C / H D L-C$ & $5.4 \pm 1.5$ & $5.2 \pm 1.3 * *$ & $4.4 \pm 1.0 * *$ & $3.9 \pm 0.9 * *$ & $3.7 \pm 0.8 * *$ \\
\hline Triglyceride $(\mathrm{mg} / \mathrm{dl})$ & $176.9 \pm 80.7$ & $146.8 \pm 57.0 * *$ & $134.6 \pm 62.8 * *$ & $137.8 \pm 106.3 *$ & $131.0 \pm 87.0^{* *}$ \\
\hline \multicolumn{6}{|l|}{ 4-week rehabilitation } \\
\hline$T C(m g / d l)$ & $189.0 \pm 30.4$ & $N D$ & $184.9 \pm 29.8$ & $187.2 \pm 27.7$ & $192.2 \pm 25.8$ \\
\hline$H D L-C(m g / d l)$ & $39.2 \pm 8.8$ & $N D$ & $41.9 \pm 10.2$ & $56.3 \pm 11.9 * *$ & $66.2 \pm 11.7 * *$ \\
\hline$L D L-C(m g / d l)$ & $126.2 \pm 33.7$ & $N D$ & $119.1 \pm 32.4$ & $106.5 \pm 27.4 *$ & $106.6 \pm 27.6$ \\
\hline$T C / H D L-C$ & $5.0 \pm 1.3$ & $N D$ & $4.6 \pm 1.3 *$ & $3.5 \pm 0.8^{* *}$ & $3.0 \pm 0.9 * *$ \\
\hline Triglyceride $(\mathrm{mg} / \mathrm{dl})$ & $145.3 \pm 57.1$ & $N D$ & $134.0 \pm 54.3$ & $122.4 \pm 53.2 *$ & $96.8 \pm 42.2 * *$ \\
\hline
\end{tabular}

Data are expressed as mean $\pm S D .{ }^{*} p<0.05, * * p<0.01$ vs baseline. TC, total-cholesterol; HDL-C, high-density lipoprotein cholesterol; $L D L-C$, low-density lipoprotein cholesterol; $N D$, not determined.

the ratio of TC/HDL-C had decreased significantly at the 1-, 6- and 12-month follow-ups in both groups (Table2). When those patients in the 2-week rehabilitation group who were not taking lipid-lowering agents were analyzed separately, the ratio of TC/HDL-C was still decreased significantly $(\mathrm{n}=30$, baseline vs 1 -month, 6-month and 12-month, $\mathrm{p}<0.05$ for all).

\section{Exercise Frequency}

At the baseline in the 2-week rehabilitation group, only $14 \%$ patients had regular physical activity, $29 \%$ patients had low physical activity and $57 \%$ patients were sedentary. In the control group, the proportions were $21 \%, 18 \%$ and $61 \%$, respectively. There was no significant difference in the frequency of habitual exercise among the 3 groups at 
Table 3 Scores for State-Trait Anxiety and Self-Rating Questionnaire for Depression at the Baseline, 1-Month, 6-Month and 12-Month Follow-ups in the Rehabilitation Groups and the Control Group

\begin{tabular}{lcccc}
\hline \hline & Baseline & 1 -month & 6 -month & 12-month \\
\hline 2-week rehabilitation & & & & \\
STAI-I & $44.2 \pm 10.5$ & $37.6 \pm 10.0^{* *}$ & $34.2 \pm 9.1^{* *}$ & $35.4 \pm 12.4^{*}$ \\
STAI-II & $40.7 \pm 8.3$ & $39.2 \pm 8.9$ & $35.3 \pm 7.6^{* *}$ & $38.9 \pm 11.2$ \\
SRQ-D & $8.1 \pm 3.4$ & $7.9 \pm 4.1$ & $6.8 \pm 2.9$ & $7.7 \pm 5.2$ \\
4-week rehabilitation & & & & \\
STAI-I & $42.6 \pm 9.6$ & $37.4 \pm 9.6^{*}$ & $38.6 \pm 13.7^{*}$ & $40.6 \pm 9.7$ \\
STAI-II & $43.6 \pm 11.0$ & $40.8 \pm 11.0^{*}$ & $42.2 \pm 11.9$ & $43.7 \pm 11.0$ \\
SRQ-D & $9.5 \pm 4.2$ & $8.6 \pm 4.0$ & $7.3 \pm 4.2^{*}$ & $9.2 \pm 3.8$ \\
Control & & & & \\
STAI-I & $40.4 \pm 8.0$ & $N D$ & $40.0 \pm 11.8$ & $37.7 \pm 10.7$ \\
STAI-II & $41.6 \pm 11.4$ & $N D$ & $42.1 \pm 11.4$ & $39.5 \pm 12.4$ \\
SRQ-D & $7.1 \pm 4.4$ & $N D$ & $6.9 \pm 4.6$ & $7.2 \pm 3.9$ \\
\hline
\end{tabular}

Data are expressed as mean $\pm S D .{ }^{*} p<0.05$, ${ }^{* *} p<0.01$ vs data of the baseline. STAI-I, state anxiety score; STAI-II, trait anxiety score; $S R Q-D$, self-rating questionnaire for depression; $N D$, not determined.

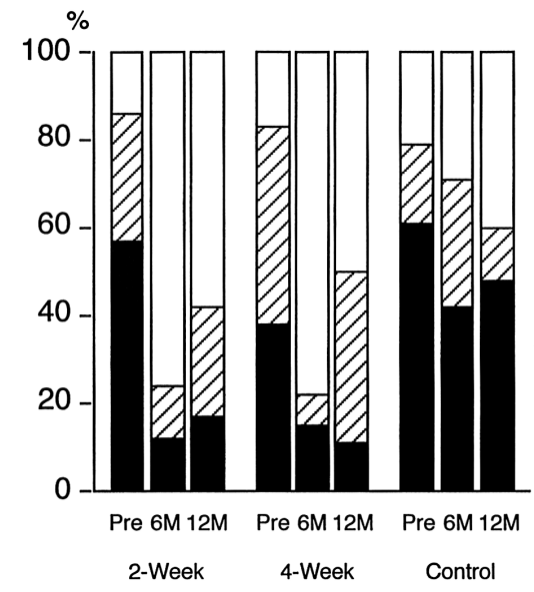

Fig 3. Changes in exercise habits up to 12 months after the rehabilitation program. ( $\mathbf{\square})$ sedentary group (no physical activity); ( $\mathbb{Z}$ ) low physical activity group $(<2$ times per week); $(\square)$ regular physical activity group (physical activity $\geq 2$ times per week). 4-week, 4-week rehabilitation group; 2-week, 2-week rehabilitation group; Control, control group. Data are expressed as percentage.

the baseline. At the 6-month follow-up, more than $75 \%$ patients had regular physical activity in both the rehabilitation groups. In contrast, only $21 \%$ patients had regular physical activity in the control group. At the 12-month follow-up, regular physical activity was being maintained in both the rehabilitation groups (Fig 3).

\section{STAI}

In both the rehabilitation groups, the STAI-I score was improved significantly after completion of the rehabilitation program, and at the 6- and 12-month follow-ups compared with the baseline. In contrast, it was unchanged in the control group at the 6- and 12-month follow-ups (Table 3). When the STAI-I score was analyzed in patients who received all the consecutive evaluations, it was improved in the 2-week rehabilitation group compared with the control group (repeated ANOVA, $\mathrm{p}=0.0095$ ).

The STAI-II score improved significantly after completion of the rehabilitation in the 4-week rehabilitation group, and had improved significantly at the 6-month follow-up in the 2-week rehabilitation group. However, there was no improvement in the score in the control group during the
A

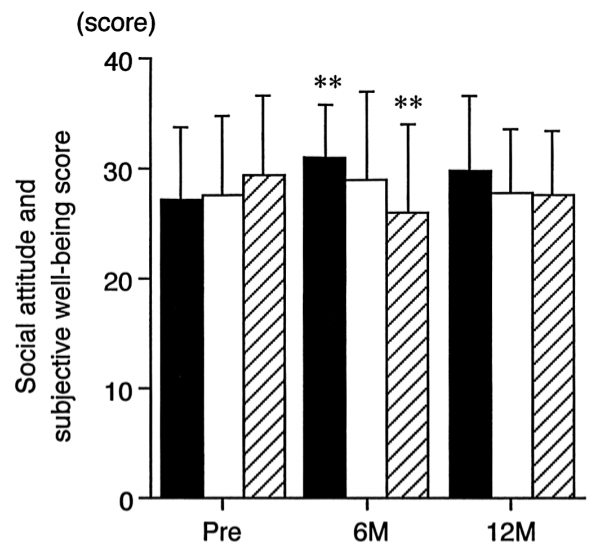

B

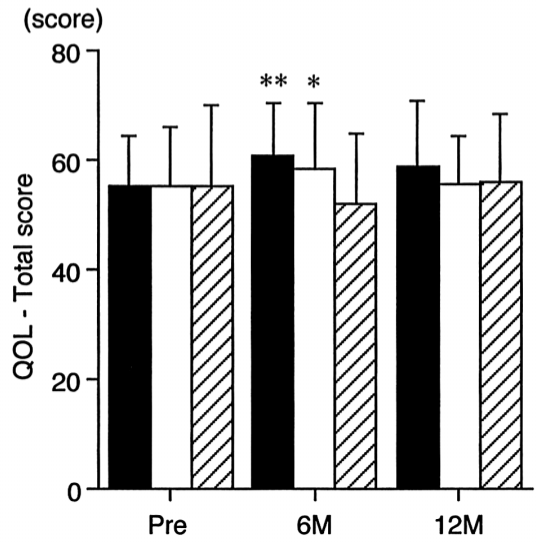

Fig 4. Quality of life (QOL) scores in 2-week rehabilitation group

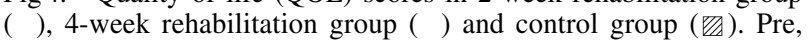
before rehabilitation; $6 \mathrm{M}, 6$ months after the rehabilitation; $12 \mathrm{M}, 12$ months after the rehabilitation. Data are expressed as mean \pm SD. ${ }^{*} \mathrm{p}<0.05, * * \mathrm{p}<0.01$ vs Pre.

follow-up period (Table3).

$S R Q-D$

The depression score in the 2-week rehabilitation group had not changed at the 6- and 12-month follow-ups. Before entry into the program, the SRQ-D depression score in the 4-week rehabilitation group was significantly higher than in 
both the 2-week rehabilitation group and the control group. At the 6-month follow-up, the score had improved significantly only in the 4-week rehabilitation group (Table 3).

\section{$Q O L$}

In the 2-week rehabilitation group, the social attitude and subjective well-being score and the QOL total score had improved significantly at the 6-month follow-up, but in the control group, the social attitude and subjective wellbeing score decreased significantly (Fig 4). At the 12-month follow-up, however, there were no significant differences in the social attitude and subjective well-being score and the QOL total score between the rehabilitation groups and the control group. When the social attitude and subjective well-being score was analyzed in patients who received all consecutive evaluations, it was improved in the 2-week rehabilitation group compared with the control group (repeated-ANOVA, $\mathrm{p}=0.001$ ).

\section{Discussion}

Cardiac rehabilitation aims to return patients to an active and productive life. We have demonstrated physical and psychological improvements after the hospitalized 2-week phase II cardiac rehabilitation program and these improvements were maintained at the 12-month follow-up.

In the 2-week rehabilitation group, the peak $\dot{\mathrm{VO}}_{2}$ and AT had increased at the 6-month follow-up compared with 1 month after completion of the program and may reflect the fact that more than $70 \%$ of patients in the rehabilitation group continued regular physical activity up to the 6-month follow-up. The physical exercise program consisted of $60-80 \mathrm{~min}$ of aerobic exercise under the supervision of a physiotherapist and 20-30 min of self-monitored walking each day. The patients were encouraged to undertake exercise activities that were personally enjoyable and could be sustained long-term after their discharge from hospital. Physical training equal to or more than twice per week generally produces meaningful increases in the maximal $\dot{\mathrm{VO}}_{2}{ }^{17,18}$ and reduces the prevalence of fatty liver. ${ }^{19}$ Although the exercise frequency was similar in both the rehabilitation groups, a significant improvement in exercise tolerance was observed only in the 2-week rehabilitation group, possibly because that group was younger than the 4-week rehabilitation group and the patients in the latter group may have fully recovered their physical capacity at the end of the 4-week rehabilitation program. Patients and their spouses had information sessions twice a year (6- and 12-month follow-ups) to ascertain individual exercise intensity and frequency and it may be that the intensive and self-monitored nature of the program is the reason for the patients' high level of motivation.

It has been reported that the most favorable impact on lipid levels is from multifactorial rehabilitation programs that include exercise training, dietary education, counselling, psychological support and pharmacological treatment 20 Lipid lowering therapies with statins have been reported to result in stabilization of atherosclerotic plaques. ${ }^{21-23}$ In the present study, some patients were taking lipid-lowering agents and continued to take them without change throughout the trial. In the 2-week rehabilitation group, serum concentrations of HDL-C and TG improved just after completion of the program and at the 1-month follow-up and, moreover, had improved significantly at the 6-month and even 12-month follow-ups. Most randomized controlled trial studies have reported that cardiac rehabilitation had beneficial effects on the levels of TC, HDL-C and TG? ${ }^{24}$ The Framingham study reported that a TC/HDL-C ratio greater than 4.5 was strongly associated with coronary heart disease ${ }^{25,26}$ Exercise training can significantly reduce this ratio and potentially reduce the risk of subsequent cardiovascular events in patients with coronary heart disease 27 The present study found that the TC/HDL-C ratio deceased to less than 4.5 at the 6-month and even at the 12-month follow-ups. Exercise has been shown to increase serum HDL-C, and consequently may reduce the risk of further development of atherosclerotic lesions. These findings support that multifactorial intervention is effective in controlling serum lipid profiles and taken together, the present results support our belief that patients with MI should be routinely referred to and encouraged to take part in phase II cardiac rehabilitation.

Although the psychosocial findings of the present study are limited by the protocol used, the 2-week cardiac rehabilitation program resulted in an improved psychosocial status at the 6-month follow-up. Many patients suffer from reactive anxiety and depression following acute MI and those with poor psychosocial adaptation often experience social isolation, low compliance of medical and exercise regimens and unrealistically negative health perceptions ${ }^{28}$ Improvement in the patient's perception of mood is an important outcome of cardiac rehabilitation as it is well known that psychological problems have a major impact on recovery from major cardiovascular events. Exercise training in cardiac rehabilitation has been proven to decrease the negative psychological impact of $\mathrm{MI}^{29}$ and phase II cardiac rehabilitation will reassure patients that they can return to an independent and active lifestyle with fewer problems. In the 2-week rehabilitation group, improvements in psychological status and health-related QOL were observed at the 1-month and even at the 6-month follow-up, which indicates that in this regard the 2-week rehabilitation program has the same benefit as the 4-week rehabilitation program. At the 12-month follow-up, however, the scores for social attitude and subjective wellbeing and QOL were similar in the rehabilitation groups and the control group, which might reflect the natural recovery course of acute MI. It has been reported that patients with no source of emotional support tended to be admitted with less clinically severe disease and that the association with adverse outcomes was not apparent until several months after discharge $3^{30}$

\section{Study Limitations}

The present study was not a randomized clinical study and we did not measure exercise tolerance or serum lipid concentrations in the control group. Furthermore, levels of motivation in the rehabilitation group might have been higher than those in the control group. The patients who participated in the program may have been more concerned about their recovery from illness.

\section{Cost-Benefit}

An evaluation of the cost-benefit of our new 2-week hospitalized program of phase II cardiac rehabilitation revealed that the average inclusive cost is $¥ 60,000-90,000$ per person, which may not be sufficient to pay for occupation of one bed for 2 weeks. $3{ }^{1}$ In Japan, almost everyone has to enrol in public health insurance, which covers $70-80 \%$ of hospital charges such as operations and laboratory examinations. Many people also enrol in private insurance, which 
covers hospital charges and extra charges associated with hospitalization. An economic evaluation should be a comparative analysis of alternative courses of action in terms of both costs and consequences; this was not done in the present study. Further studies are required to evaluate the cost-benefit and to clarify the length of program that is most cost-effective.

\section{Conclusion}

Our new 2-week hospitalized program of phase II cardiac rehabilitation improved the management of hyperlipidemia, frequency of physical exercise and psychological status in patients with MI, with the improvements continuing for up to 12 months after the rehabilitation program. Therefore, this program may contribute to the secondary prevention of MI as well as to the recovery of the physical and psychological activities of patients.

\section{References}

1. Wenger NK, Froelicher ES, Smith LK, Ades PA, Berra K, Blumenthal JA, et al: Cardiac Rehabilitation: Clinical Practice Guideline No. 17. Rockville, MD: U.S. Department of Health and Human Services, Agency for Health Care Policy and Research and the National Heart, Lung, and Blood Institute. AHCPR Publication No.96-0672 October 1995: $1-26$

2. Mark DB, Naylor CD, Phil D, Hlatky MA, Califf RM, Topol EJ, et al: Use of medical resources and quality of life after acute myocardial infarction in Canada and The United States. N Engl J Med 1994; 331: $1130-1135$

3. Pearson TA, McBride PE, Miller NH, Smith SC: Organization of preventive cardiology service (Task Force 8 of the 27th Bethesda Conference). J Am Coll Cardiol 1996; 27: 1039-1047

4. Linxue L, Nohara R, Makita S, Hosokawa R, Hata T, Okuda K, et al: Effect of long-term exercise training on regional myocardial perfusion changes in patients with coronary artery disease. Jpn Circ J 1999; 63: $73-78$

5. Saito M: Cardiac rehabilitation in Europe, USA and Japan. Shinzo Rehabil 1999; 4: 37-39 (in Japanese)

6. Yoshida T, Kohzuki M, Yoshida K, Hiwatari M, Kamimoto M, Yamamoto C, et al: Physical and psychological improvement after phase II cardiac rehabilitation in patients with myocardial infarction. Nurs Health Sci 1999; 1: 163-170

7. Zavala DC: Metabolic Testing. In: Pashkow FJ, Dafoe WA, editors. Clinical cardiac rehabilitation: A cardiologist's guide. Maryland: Williams \& Wilkins, 1993: 78-101

8. Samuels B, Schumann J, Kiat H, Friedman J, Berman DS: Acute stent thrombosis associated with exercise testing after successful percutaneous transluminal coronary angioplasty. Am Heart J 1995; 130: $1120-1122$

9. Wasserman K, Whipp BJ: Exercise physiology in health and disease. Am Rev Respir Dis 1975; 112: 219-249

10. Wasserman K: The anaerobic threshold measurement to evaluate exercise performance. Am Rev Respir Dis 1984; 129(Suppl): S35-S40

11. Friedewald WT, Levy RI, Fredrickson DS: Estimation of the concentration of low-density lipoprotein cholesterol in plasma, without use of the preparative ultracentrifuge. Clin Chem 1972; 18: 499-502
12. Spielberger CD, Gorsuch RL, Lushene RE: Manual for the state-trait anxiety inventory. PaloAlto: Consulting Psychologists Press, 1970

13. Shumaker SA, Anderson RT, Czajkowski SM: Psychological tests and scales. In: Spilker B, editor. Quality of life assessments in clinical trials. New York: Raven Press, 1990: 95-113

14. Tsutsui S: Medical approaches on psychosomatic stress. Tokyo: Sindan to Chiryo-sya, 1993: 104-106 (in Japanese)

15. Rockliff BW: A brief self-rating questionnaire for depression (SRQD). Psychosomatics 1969; 10: 236-243

16. Kayaba K, Nagashima K, Saito M, Omae T, Seki A, Arakawa K, et al: The development of quality of life scales for Japanese patients with cardiovascular diseases. J Jpn Assoc Cerebro-Cardiovasc Dis Cont 1990; 25: 89-96

17. Wenger HA, Bell GJ: The interactions of intensity, frequency, and duration of exercise training in altering cardiorespiratory fitness. Sports Med 1986; 3: 346-356

18. American College of Sports Medicine Position Stand: The recommended quantity and quality of exercise for developing and maintaining cardiorespiratory and muscular fitness in healthy adults. Med Sci Sports Exerc 1990; 22: 265-274

19. Hsieh SD, Yoshinaga H, Muto T, Sakurai Y: Regular physical activity and coronary risk factors in Japanese man. Circulation 1998; 97: 661-665

20. Ornish D, Brown SE, Scherwitz LW, Billings LW, Armstrong WT, Ports TA, et al: Can lifestyle changes reverse coronary heart disease? The Lifestyle Heart Trial. Lancet 1990; 336: 129-133

21. Sacks FM, Pfeffer MA, Moye LA, Rouleau JL, Rutherford JD, Cole TG, et al: The effect of pravastatin on coronary events after myocardial infarction in patients with average cholesterol levels. $N$ Engl J Med 1996; 335: $1001-1009$

22. The Scandinavian Simvastatin Survival Study Group: Randomized trial of cholesterol lowering in 4444 patients with coronary heart disease. The Scandinavian simvastatin survival study $(4 \mathrm{~S})$. Lancet 1997; 344: 1383-1389

23. Shepherd J, Cobbe SM, Ford I, Isles CG, Lorimer AR, Macfarlane $\mathrm{PW}$, et al: Prevention of coronary heart disease with pravastatin in men with hypercholesterolemia. $N$ Engl J Med 1995; 333: 1301-1307

24. Schuler G, Hambrecht R, Schlierf G, Niebauer J, Hauer K, Neumann $\mathrm{J}$, et al: Regular physical exercise and low-fat diet: Effects of progression of coronary artery disease. Circulation 1992; 86: 1-11

25. Castelli WP, Anderson K: A population at risk: Prevalence of high cholesterol levels in hypertensive patients in the Framingham Study. Am J Med 1986; 80(Suppl2A): 23-32

26. Wilson PW, Garrison RJ, Castelli WP, Feinleib M, Mcnamara PM, Kannel WB, et al: Prevalence of coronary heart disease in the Framingham Offspring Study: Role of lipoprotein cholesterols. Am J Cardiol 1980; 46: 649-654

27. Engblom E, Ronnemaa T, Hamalainen H, Kallio V, Vanttinen E, Kunts LR: Coronary heart disease risk factors before and after bypass surgery: Results of a controlled trial on multifactorial rehabilitation. Eur Heart J 1992; 13: 232-237

28. Moser DK, Dracup K: Psychological recovery from a cardiac event: The influence of perceived control. Heart Lung 1995; 24: 273-280

29. Milani RV, Lavie CJ, Cassidy MM: Effects of cardiac rehabilitation and exercise training programs on depression in patients after major coronary events. Am Heart J 1996; 132: 726-732

30. Berkman LF, Summers LL, Horwiz RI: Emotional support and survival after myocardial infarction: A prospective, population-based study of the elderly. Ann Intern Med 1992; 117: 1003-1009

31. Insurance Office of the Ministry of Health and Welfare of Japan: The guidelines of medical expenses. Tokyo: The Research Institute of Social Insurance, 1996 (in Japanese) 